\title{
Erratum
}

Mohammed H. Aljahdali and Mohamed Elhag

\section{Erratum to "Calibration of the depth invariant algorithm to monitor the tidal action of Rabigh City at the Red Sea Coast, Saudi Arabia"}

https://doi.org/10.1515/geo-2020-0236

received February 19, 2021; accepted February 19, 2021

In the published article "Aljahdali MH, Elhag M. Calibration of the depth invariant algorithm to monitor the tidal action of Rabigh City at the Red Sea Coast, Saudi Arabia. Open Geosci. 2020;12(1):1666-78. doi: 10.1515/geo-2020-0217," the original version of the article contained an error in the grant number listed in the Acknowledgements.
The Acknowledgements should be corrected as follows:

This project was funded by the Deanship of Scientific Research (DSR), King Abdulaziz University, Jeddah (Grant No. G-90-150-1441). The authors, therefore, acknowledge with thanks DSR technical and financial support.

The authors apologize for any inconvenience that it may have caused. 